Supporting Information

\title{
Aerophilic Co-Embedded N-Doped Carbon Nanotube Arrays as Highly Efficient Cathodes for Aluminum-Air Batteries
}

Shuhui Liu ${ }^{a, b}$, Zibo Cao ${ }^{a, b}$, Yu Meng ${ }^{a, b}$, Yajie Li ${ }^{a, b}$, Weimin Yang ${ }^{c}$, Zheng Chang, ${ }^{a, b, *}$ Wen Liu ${ }^{a, b, *}$, Xiaoming Sun ${ }^{a, b}$

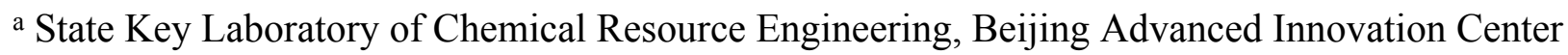
for Soft Matter Science and Engineering, Beijing University of Chemical Technology, Beijing, China 100029.

${ }^{\mathrm{b}}$ School of Chemistry, Beijing University of Chemical Technology, Beijing, China 100029.

${ }^{\mathrm{c}}$ College of Mechanical and Electrical Engineering, Beijing University of Chemical Technology, Beijing, China 100029.

Corresponding Authors:

*Zheng Chang- changzheng@mail.buct.edu.cn

*Wen Liu-wenliu@mail.buct.edu.cn 

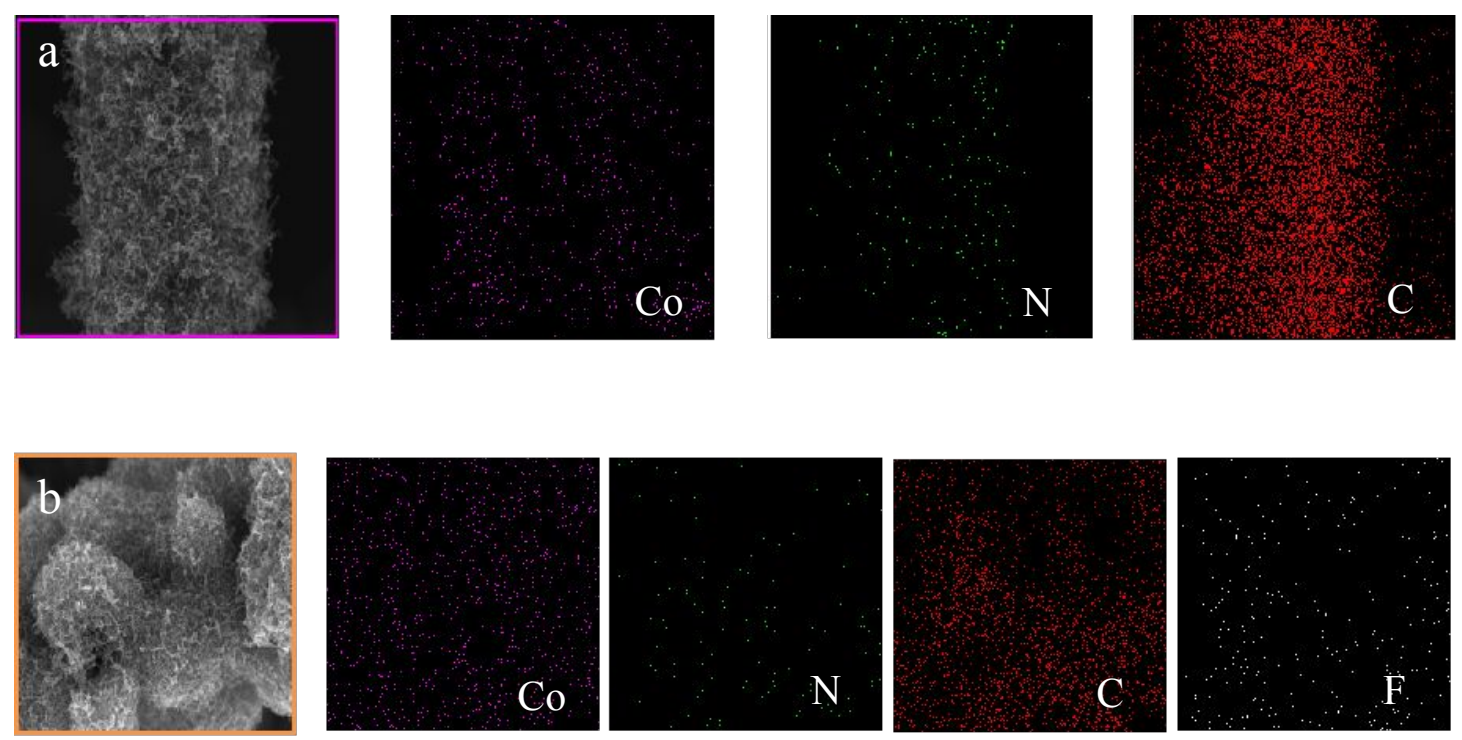

Figure S1. Elemental mappings of CoNCNTs/CP (scale bar: $1 \mu \mathrm{m}$ ) (a) before and (b) after PTFE modification. 


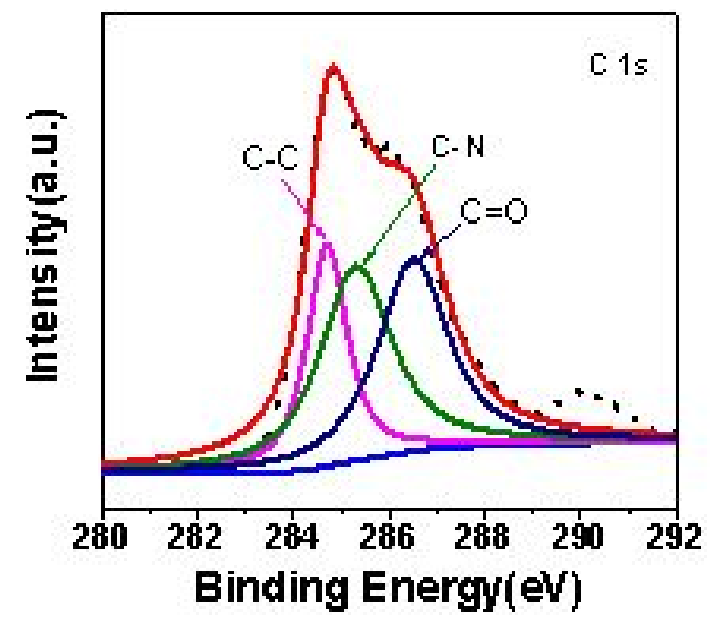

Figure S2. C 1s XPS spectrum of CoNCNTs/CP. 

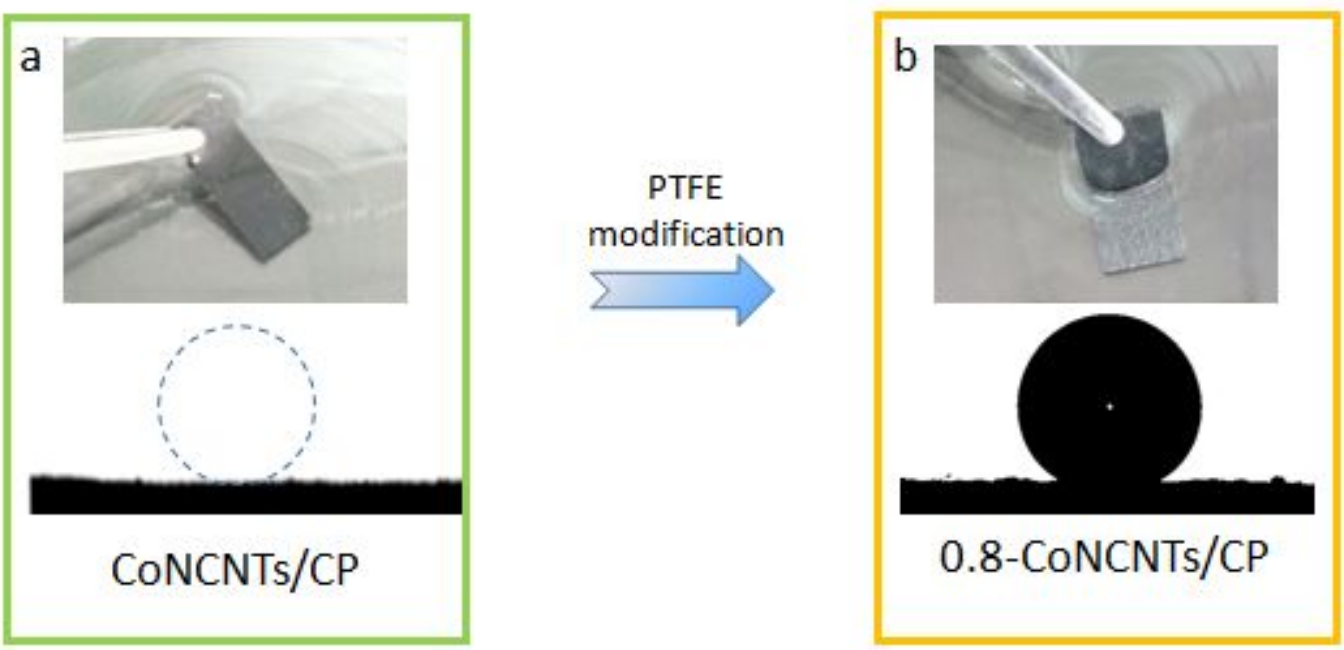

Figure S3. Basic solution contact angle results on CoNCNTs/CP (a) before and (b) after PTFE modification. 

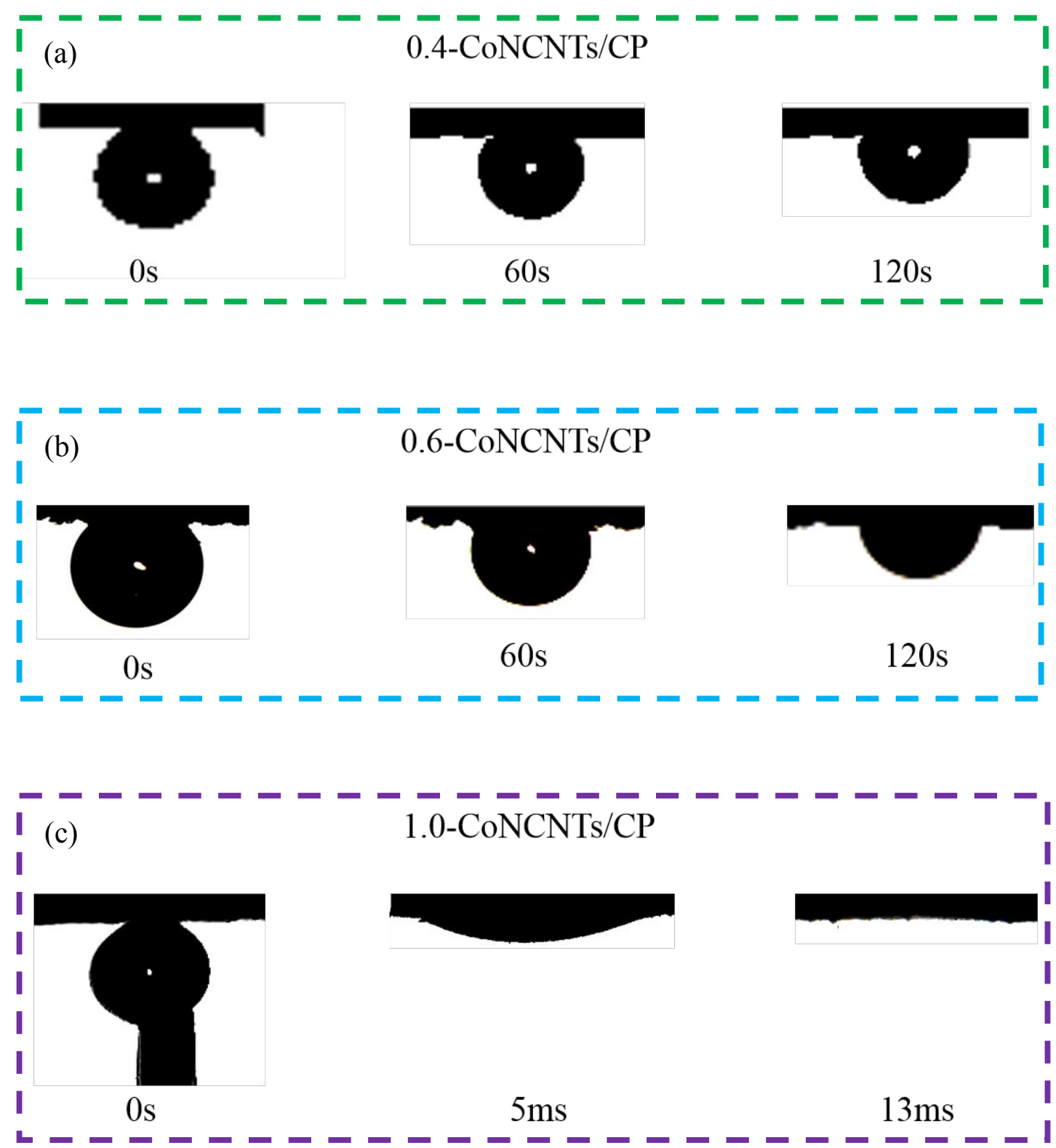

Figure S4. Basic bubble contact angle results on (a) $0.4-\mathrm{CoNCNTs} / \mathrm{CP}$; (b) $0.6-$ CoNCNTs/CP and (c) 1.0-CoNCNTs/CP. 


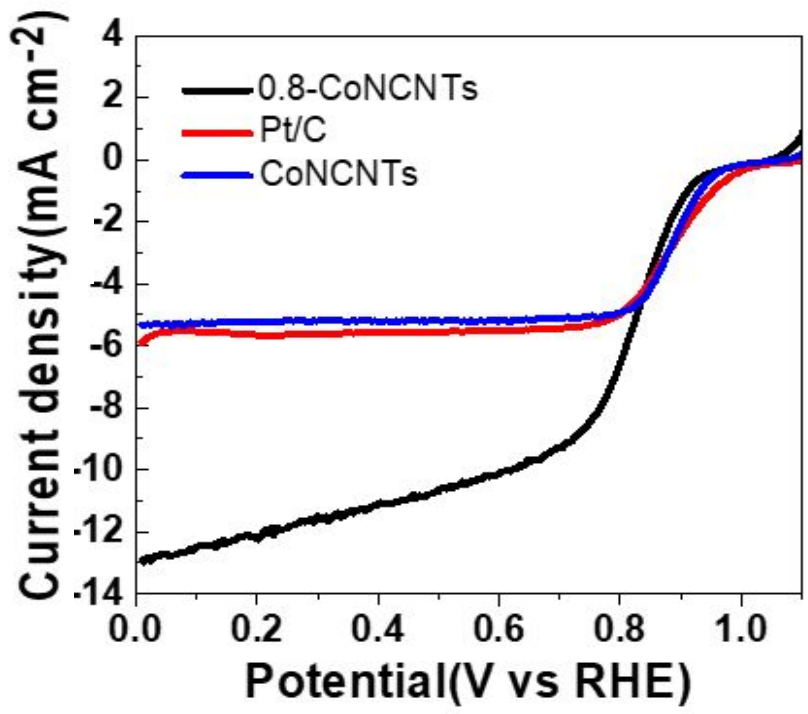

Figure S5. ORR polarization curves of $0.8-\mathrm{CoNCNTs}, \mathrm{Pt} / \mathrm{C}$ and CoNCNTs with a rotating rate of $1600 \mathrm{rpm}$ at a scan rate of $5 \mathrm{mV} \cdot \mathrm{s}^{-1}$ in an oxygen-saturated $0.1 \mathrm{M}$ $\mathrm{KOH}$ electrolyte. 
a
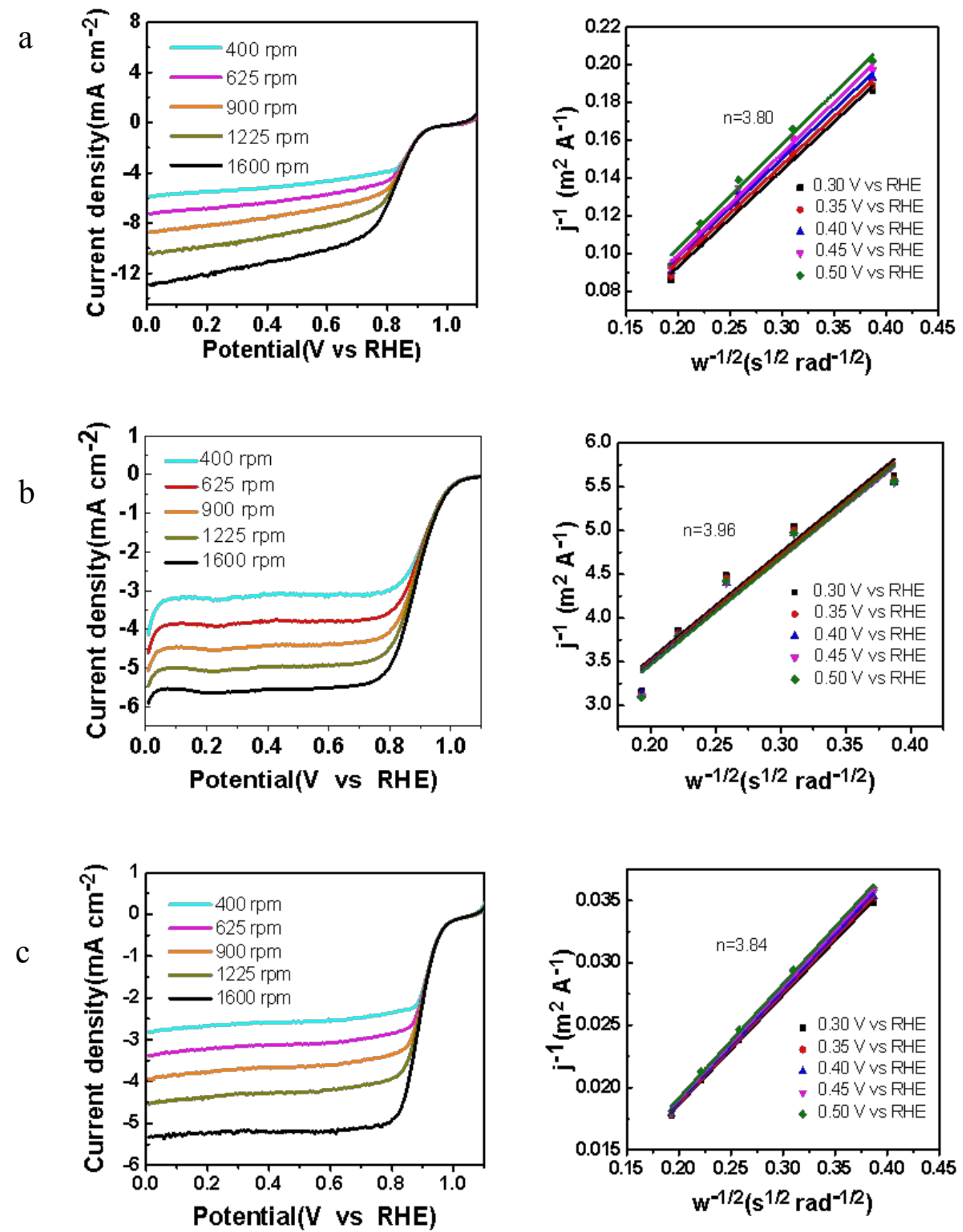

Figure S6. ORR polarization curves recorded at different rotation speeds with the corresponding K-L plots of (a) 0.8-CoNCNTs; (b) Pt/C and (c) CoNCNTs in an oxygen-saturated $0.1 \mathrm{M} \mathrm{KOH}$ electrolyte. 

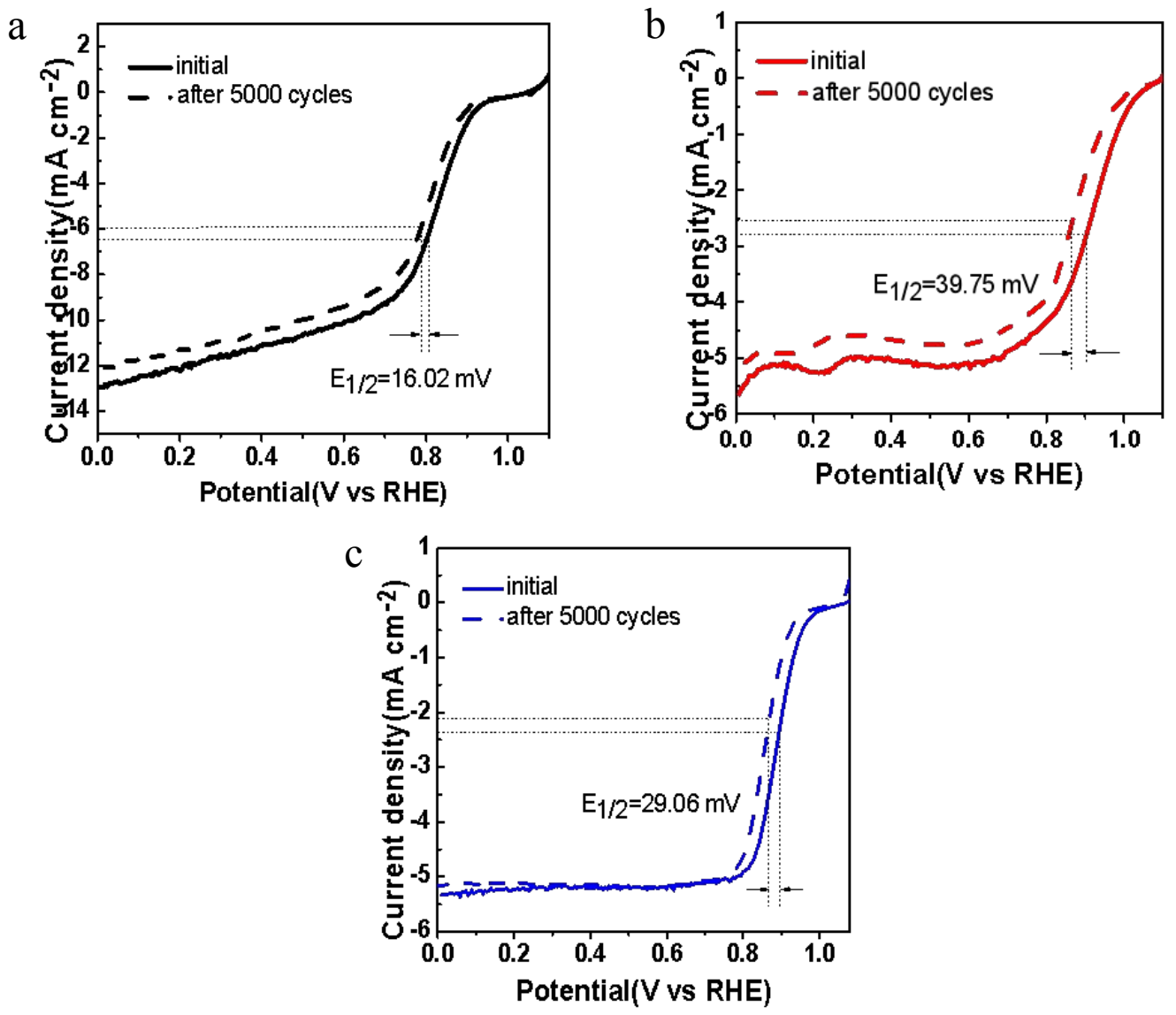

Figure S7. ORR polarization curves of (a) 0.8-CoNCNTs; (b) Pt/C and (c) CoNCNTs before and after 5000 cycles at a scan rate of $1000 \mathrm{mV} \cdot \mathrm{s}^{-1}$ in an oxygen-saturated 0.1 M KOH electrolyte. 

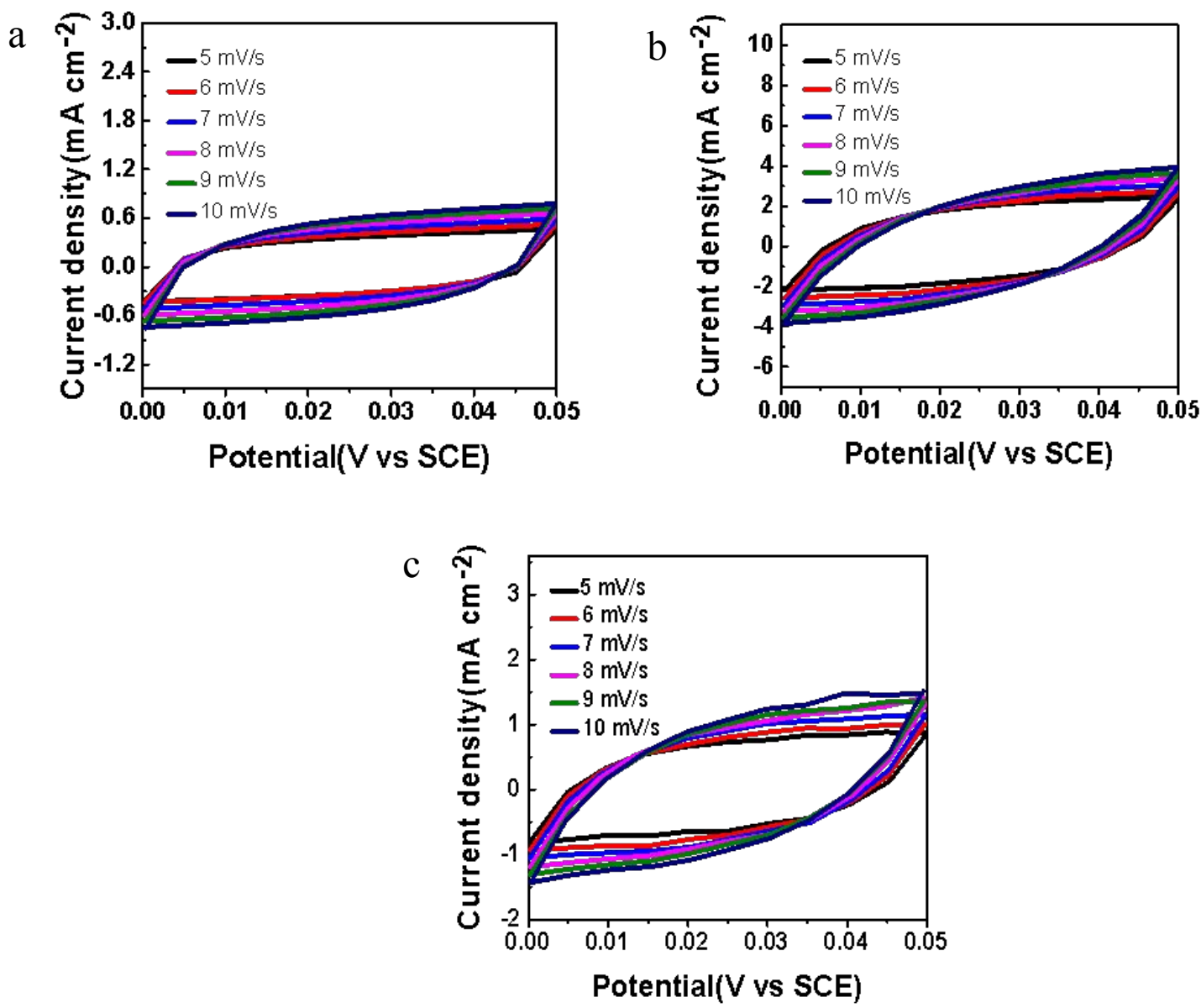

Figure S8. ECSA measurements of (a) $\mathrm{Pt} / \mathrm{C}$; (b) CoNCNTs/CP and (c) 0.8 CoNCNTs/CP by measuring CV curves at $0 \sim 0.05 \mathrm{~V}$ (vs. SCE) at different scan rates. 


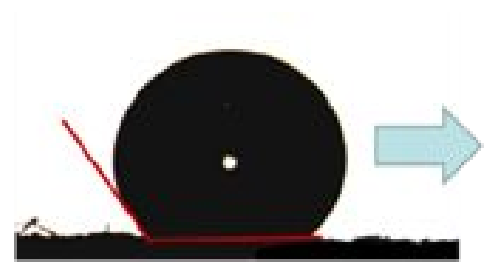

Os, $130^{\circ}$

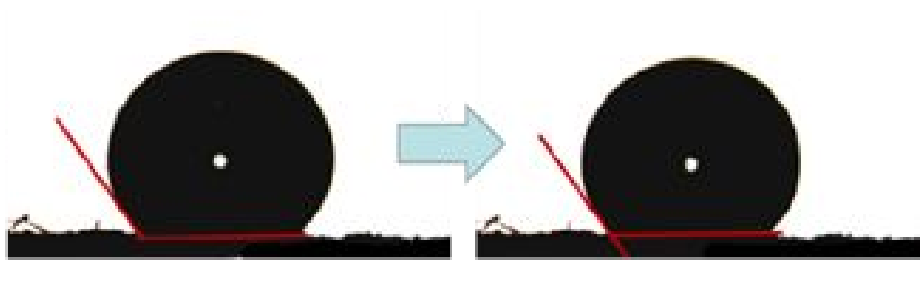

$180 \mathrm{~s}, 128^{\circ}$

$360 \mathrm{~s}, 127^{\circ}$

Figure S9. Electrolyte droplet contact angles on 0.8 -CoNCNTs/CP after long-term reaction in $6 \mathrm{M} \mathrm{KOH}$ electrolyte. 

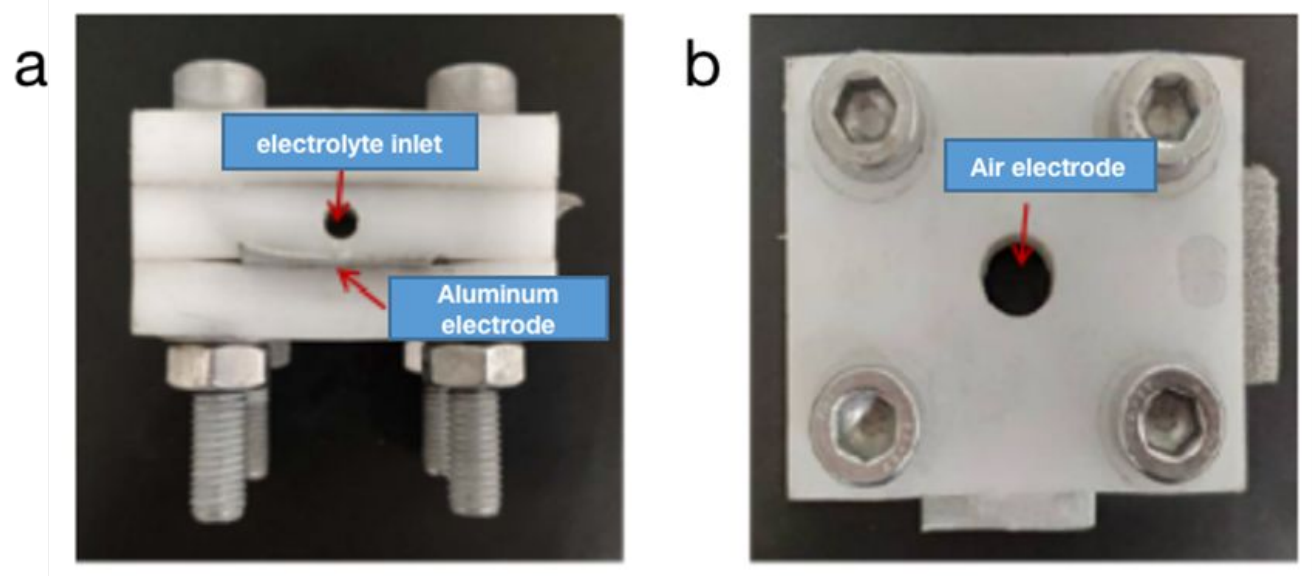

Figure S10. Optical pictures on (a) the side and (b) the front of the self-assembled Aluminum-air battery. 


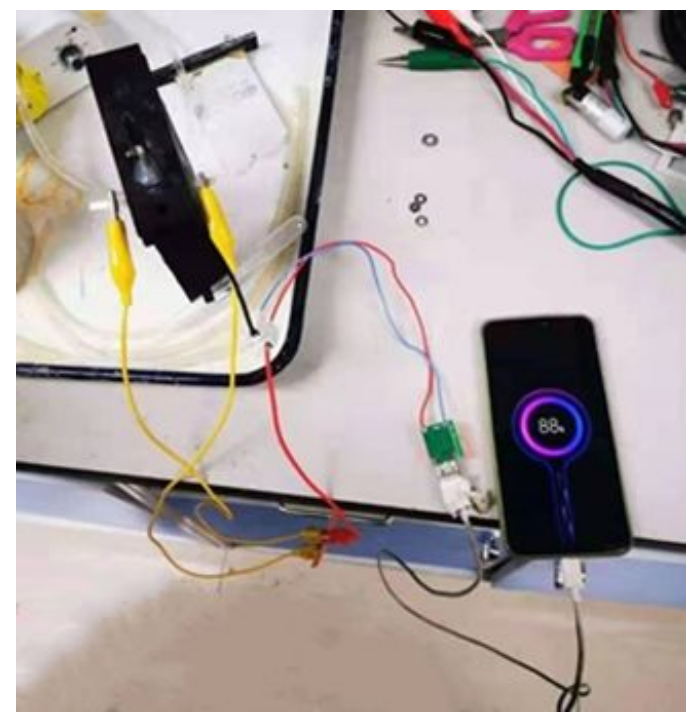

Figure S11. Possible application demonstration: charging a mobile phone with a prismatic Aluminum-air battery. 
Table S1. The relative atomic contents of Co, N, C and O of CoNCNTs/CP according to the XPS result.

\begin{tabular}{|c|c|}
\hline Element & Atomic\% \\
\hline Co & 1.09 \\
\hline $\mathrm{N}$ & 7.15 \\
\hline $\mathrm{C}$ & 88.24 \\
\hline $\mathrm{O}$ & 3.52 \\
\hline
\end{tabular}

